\title{
Influencia de los trastornos de la personalidad en la eficacia en el tratamiento de los trastornos adaptativos: un estudio preliminar
}

\author{
CRISTINA SANAHUJA LEIRA \\ al260571@uji.es \\ IRYNA RACHYLA \\ rachyla@uji.es \\ MAR MolÉs AMPOSTA \\ molesm@uji.es \\ Soledad Quero Castellano \\ squero@uji.es
}

\section{Resumen}

Introducción: Los pocos estudios llevados a cabo ponen de manifiesto que los trastornos adaptativos (TA) presentan una alta comorbilidad con los trastornos de la personalidad (TP) (Doherty y cols., 2014). Se sugiere que esto influye en la respuesta al tratamiento del TA. El objetivo de este trabajo es analizar si existen diferencias en la eficacia del tratamiento entre pacientes con TA y comorbilidad con TP o rasgos patológicos y pacientes que solamente presenten TA. Método: La muestra incluyó 97 participantes: 55 con TA y comorbilidad con TP o rasgos de personalidad patológica y 42 sin TP. Los participantes recibieron un protocolo de tratamiento que incluía técnicas cognitivo-conductuales (TCC) y estrategias de psicología positiva en dos formas: tradicional o apoyado en realidad virtual (RV). Como medidas de eficacia, se calcularon los índices de cambio fiable obtenidos tras el tratamiento y seguimiento a los tres meses para el inventario de depresión de Beck, el inventario de estrés y pérdida, el inventario de crecimiento postraumático y la escala de afecto negativo del PANAS. Resultados: No se encontraron diferencias estadísticamente significativas en ninguna de las medidas ni en el postratamiento ni en el seguimiento. Conclusiones: Los resultados preliminares obtenidos parecen indicar que la presencia de un TP o rasgos patológicos de la personalidad no influyen en la eficacia del tratamiento para los TA y tampoco en el mantenimiento de los cambios a medio plazo. Por tanto, ambos grupos parecen beneficiarse por igual de un tratamiento TCC en formato tradicional o apoyado en la RV.

Palabras clave: trastorno adaptativo, trastorno de personalidad, eficacia, TCC, psicología positiva, realidad virtual. 


\section{Abstract}

Introduction: Very few studies have been conducted to show that Adjustment Disorders (AD) have a high comorbidity with personality disorders (PD) (Doherty \& cols, 2014.). It has been suggested that this influences response to treatment for AD. The aim of this work was to analyse whether there were differences in treatment efficacy between patients with $A D$ and comorbidity with $P D$ and/or pathological traits, and the patients that present only AD. Method: The sample included 97 participants: 55 with AD and comorbility with PD or pathological personality traits, and 42 without PD. Participants received a treatment protocol that included cognitive-behavioural techniques (CBT) and positive psychology strategies in two forms: traditional or supported by virtual reality (VR). As efficacy measures the reliable change, the indices obtained at post-treatment and 3-month follow-up were calculated for the Beck Depression Inventory, the Stress and Loss Inventory, the Posttraumatic Growth Inventory, and the PANAS negative affect scale. Results: No statistically significant differences were found in any outcome measures at either post-treatment or follow-up. Conclusions: The obtained preliminary results seem to indicate that presence of PD and/or personality pathological traits affects neither AD treatment efficacy nor maintenance of short-term changes. Therefore, both groups seem to equally benefit from СВТ treatment in the traditional format or supported by VR.

Keywords: Adjustment Disorder, Personality Disorder, efficacy, CBT, positive psychology, virtual reality

\section{Introducción}

Los estudios epidemiológicos llevados a cabo hasta el momento indican que los trastornos adaptativos (TA) son uno de los problemas psicológicos más frecuentes en los contextos de atención primaria y hospitalaria (por ejemplo, Carta, Balestrieri, Murru y Carolina-Hardoy, 2009). El DSM-V define los TA como una reacción emocional y comportamental negativa que interfiere en distintas áreas de la vida de la persona y aparece en respuesta a un evento estresante identificable dentro de los tres meses siguientes a la aparición de este (APA, 2013). Los clasifica en el capítulo "Trastornos relacionados con traumas y factores de estrés». Así pues, se trata de un trastorno con un gran impacto negativo en la vida de la persona que lo sufre así como un grave problema de salud que se asocia a un importante deterioro en las áreas social y laboral como, por ejemplo, un alto porcentaje de bajas laborales (Calvo, 2009). Además, se trata de un trastorno en el que existe un alto porcentaje de riesgo de suicidio (Cassey, Jabbar, O'Leary y Doherty, 2015).

Por lo que se refiere al abordaje terapéutico de los TA, la escasa atención e interés por parte de los investigadores ha llevado a que contemos con muy pocos estudios realizados acerca de su tratamiento (Casey, Dowrick y Wilkinson, 2001). Además, los pocos estudios publicados que utilizan diseños entregrupos presentan limitaciones metodológicas importantes (por ejemplo, Ben-Itzhak y cols., 2012; Hsiao y cols., 2014; Srivastava, Talukdar y Lahan, 2011). Por lo tanto, actualmente no existe un tratamiento de elección basado en la evidencia para los TA. En este sentido, nuestro grupo de investigación ha diseñado y desarrollado un protocolo de tratamiento para los TA (Botella, Baños y Guillén, 2008) que incluye técnicas de 
terapia cognitivo-comportamental (TCC) y estrategias de psicología positiva y que, además, se apoya en el uso de la realidad virtual (RV). Este protocolo para los TA ha mostrado su eficacia en diversos estudios (Andreu-Mateu, Botella, Quero, Guillén y Baños, 2012; Baños y cols., 2008; Botella, Osma, García-Palacios, Guillén y Baños, 2008; Baños y cols., 2011; Quero y cols., en prensa).

Por otro lado, los escasos estudios llevados a cabo acerca de la comorbilidad que presentan los TA indican que este trastorno presenta alta comorbilidad con los trastornos de personalidad (TP) (Doherty, Jabbar, Kelly y Casey, 2014). Algunos estudios realizados ponen de manifiesto que existen indicios de que el tratamiento de algunos trastornos del eje I se complica con la presencia de un TP (Reich y Green, 1991; Reich y Vasile, 1993). Así, estos estudios concluyen que las patologías de personalidad afectan de forma negativa al tratamiento de algunos trastornos del eje I (trastornos depresivos, trastornos de ansiedad y trastornos de abuso y dependencia de sustancias). Además, esta interferencia negativa se muestra en relación al uso de programas de TCC (Black y cols. 1994). Por lo que se refiere a los TA, no se ha encontrado en la literatura ningún estudio que explore la eficacia del tratamiento del TA cuando existe comorbilidad con un TP o con rasgos patológicos de personalidad. Sin embargo, existen estudios más recientes que exploran la eficacia del tratamiento cuando hay comorbilidad con un TP en trastornos de ansiedad (Dressen y Arntz, 1998) y en pacientes con depresión (Newton-Howes, 2008). Según Dressem y Arntz (1998), a partir de la revisión de los 15 trabajos incluidos en su estudio, no se puede concluir que, en general, la presencia de un TP afecte de forma significativa a los resultados del tratamiento para los trastornos de ansiedad (trastorno de pánico, trastorno obsesivo-compulsivo, fobia social, trastorno de ansiedad generalizada). Además, añaden que solamente se puede sospechar que algunos TP específicos, tales como el TP evitativo comórbido en pacientes con trastorno de pánico y el TP esquizotípico en pacientes con trastornos obsesivo-compulsivo, afectan negativamente al resultado del tratamiento y estos trastornos deberían investigarse más. Por su parte, en el estudio llevado a cabo por Newton-Howes (2008) en el que realiza un metanlálisis de diferentes estudios en pacientes con depresión, se concluye que presentar un TP llevaba a un peor pronóstico de la depresión, aunque no se hallaron diferencias significativas en la eficacia del tratamiento del trastorno depresivo.

El objetivo del presente estudio es explorar si existen diferencias en la eficacia del tratamiento entre pacientes con TA y comorbilidad con TP o rasgos patológicos y pacientes que solamenten presenten TA.

\section{Método}

\section{Participantes}

La muestra incluida en este estudio estaba compuesta de 97 participantes diagnosticados de TA, según el DSM-IV-TR (APA, 2000) y CIE-10 (OMS, 1992). Todos ellos se seleccionaron de entre las personas que solicitaron ayuda en el Servicio de Asistencia Psicológica de la Universitat Jaume I de Castellón (España). La gran mayoría de la muestra eran mujeres (74,2 \%). El rango de edad se situó entre los 18 y los 54 años con una media de 31,85 (DT =9,531). Respecto al estado civil, el $58,8 \%$ de la muestra estaban solteros, el $28,9 \%$ casados o conviviendo con pareja y el $12,4 \%$ estaban separados/divorciados o eran viudos. Por último, en cuanto al nivel educativo, predominaron los participantes con estudios universitarios $(74,2 \%)$, mientras que el $25,8 \%$ tenían estudios primarios o secundarios.

Por lo que se refiere al subtipo diagnóstico del TA, 15 participantes cumplian criterios para TA subtipo depresivo (15,5\%), 7 presentaron TA subtipo ansioso (7,2 \%), 73 mostraron subtipo 
mixto con ansiedad y estado de ánimo depresivo (75,3\%) y 2 cumplían criterios para el subtipo con alteración mixta de las emociones y el comportamiento $(2,1 \%)$.

Por último, en cuanto a la presencia de patología del eje II, 55 participantes se incuyeron en el grupo denominado TA con rasgos o trastorno de personalidad patológica $(56,7 \%)$, de los cuales 7 cumplían criterios para un TP (2 TP dependiente, 1 TP obsesivo-compulsivo, 1 TP depresivo, 1 TP por evitación y 2 TP paranoide) y 47, aunque no llegaban a cumplir criterios para un TP, mostraban algunos rasgos de personalidad patológica. Entre los rasgos de personalidad patológica que más abundaban en el estudio se encuentra la tendencia a la rigidez e inflexibilidad (que se llegaba a presentar en más de la mitad de los pacientes que no llegaban a cumplir criterios para un TP). El restante 43,35 ( $\mathrm{N}=42$ ) de la muestra no presentaban comorbilidad con el eje II.

\section{Medidas}

Todos los participantes pasaron por un protocolo de evaluación más amplio que incluía los instrumentos específicos para evaluar TA, así como instrumentos que recogen aspectos relacionados con el problema en concreto. A continuación se describen las medias utilizadas en este trabajo.

\section{Medidas diagnósticas}

Entrevista diagnóstica para los trastornos adaptativos (Andreu-Mateu, Botella, Baños y Quero, 2008). Consiste en una entrevista semiestructurada desarrollada por nuestro grupo de investigación para la evaluación de los TA. Para su elaboración, se tuvieron en cuenta los criterios diagnósticos tanto de la CIE-10 como del DSM-IV-TR, así como la entrevista SCID-IV y los hechos considerados relevantes en la literatura en relación a este trastorno. La entrevista incluye la exploración de la existencia de uno o varios eventos estresantes, una valoración de los síntomas presentes (evaluando su gravedad con una escala de 0 a 8 , siendo $0=$ nada y 8 = muy grave) y de las características principales de los mismos (tipo de síntomas predominantes, tiempo de latencia de aparición y duración de los síntomas, posible existencia de consecuencias en la actualidad y evolución), cuestiones que permiten realizar el diagnóstico diferencial, cuestiones que rastrean la existencia de consumo de sustancias o de condiciones físicas graves, una evaluación del malestar y la interferencia causada por el problema (mediante una adaptación de la escala de inadaptación de Echeburúa y Corral, 2000) y preguntas cualitativas sobre el acontecimiento estresante.

Entrevista clínica estructurada para los trastornos de la personalidad del eje II del DSMIV (SCID-II; First y cols., 1999). Se trata de una entrevista semiestructurada que consta de 119 preguntas (más otras adicionales para el trastorno antisocial) de respuesta abierta o cerrada. La entrevista diagnóstica recoge los criterios según la APA para los TP del cluster A (esquizoide, esquizotípico y paranoide), del cluster $B$ (antisocial, histriónico, límite y narcisista) y del cluster C (dependiente, depresivo, evitativo, obsesivo-compulsivo y pasivo-agresivo). Además de la entevista, contiene un cuestionario de personalidad autocumplimentado que actúa como cribado. Consta de 119 items de respuesta dicotómica (sí/no) y su corrección se basa en el número de respuestas afirmativas que la persona ha dado en cada uno de los posibles trastornos evaluados. En el presente estudio se ha hecho uso tanto del cuestionario como de la entrevista. En primer lugar, los participantes cumplimentaron el cuestionario y, posteriormente, en aquellos ítems contestados de forma afirmativa y que superaban la puntuación de corte, se evaluó en 
mayor profundidad administrando la parte de la entrevista correspondiente. Los estudios de fiabilidad y validez realizados muestran diferencias en los resultados, pero la mayoria informan de índices aceptables (First y cols., 1999).

\section{Medidas de eficacia}

Inventario de depresión de Beck-II (BDI-II: Beck Depression Inventory-Second Edition; Beck, Steer y Brown, 1996; validado en población española por Sanz, Navarro y Vázquez, 2003). Se trata de uno de los instrumentos más utilizados para evaluar el estado de ánimo depresivo. Consta de 21 ítems que evalúan síntomas y cogniciones relacionadas con la depresión. Cada ítem consta de 4 alternativas de respuestas ordenadas en función de la intensidad, que se evalúa en una escala de 0 a 3 . El participante debe responder como mejor describa el modo en el que se ha sentido durante las dos últimas semanas. El sumatorio de las puntuaciones permite establecer 4 rangos cuantitativos de depresión: de 0 a 9 (ausente o mínima); de 10 a 18 (entre leve y moderada); de 19 a 29 (entre moderada y grave); y de 30 a 63 (grave). En población española muestra una elevada consistenica interna tanto para la población general (coeficiente alfa de 0,87 ) como para la población clínica (coeficiente alfa de 0,89) (Sanz, Perdigon y Vázquez, 2003).

Inventario de estrés y pérdida (IEP; Quero, Mor, Molés, Baños y Botella, en preparación). Se llevó a cabo una adaptación del inventario de duelo complicado (ICG: Complicated Grief Inventory; Pirgerson y cols., 1995) con el objetivo de utilizarlo en la evaluación de situciones estresante que estén interfiriendo de forma negativa en la vida de una persona. El instrumento es igual al original con la excepción de que se sustituyeron las palabas que aluden a la persona fallecida por las que se refieren a la persona/situación que se ha perdido (por ejemplo, ruptura de pareja, cambio de trabajo...). Evalúa la intensidad con la que interfiere la situación o persona perdida en la vida del paciente en una escala de 0 (nunca) a 4 (siempre). A diferencia de la versión original del ICG, el IEP consta de 17 ítems. Datos preliminares de validación mostraron excelentes coeficientes de fiabilidad test-retest $(0,90)$ y de consistencia interna en población española tanto general $(0,91)$ como clínica con TA $(0,86)$ (Quero, Molés, Mor, Baños y Botella, 2014).

Escala de afecto positivo y negativo (Watson, Clark y Tellegen, 1988). Este cuestionario consta de 20 ítems en el que se evalúan dos dimensiones del afecto: la dimensión positiva y la negativa. Los participantes evalúan cada uno de los ítems indicando el grado en el que cada adjetivo describe mejor su estado de ánimo actual en una escala de 1 a 5 , siendo $5=$ mucho y 1 = nada o muy ligeramente. Esta escala presenta una elevada consistencia interna y una buena fiabilidad test-retest (Watson y cols., 1988). En el presente estudio solo se utilizaron los datos de los ítems de la escala de afecto negativo.

Inventario de crecimiento postraumático (PTGI: Postraumatic Growth Inventory; Tedeschi y Calhoun, 1996). Este cuestionario de 21 ítems evalúa los cambios personales positivos que ocurren tras la vivencia de una situación muy negativa o traumática. Se evalúa en un rango que va de 0 a 5 ( 0 = No experimenté este cambio como resultado de la crisis, $5=$ Experimenté este cambio en gran medida como resultado de la crisis). Puntuaciones elevadas en este inventario indican un mayor grado de crecimiento postraumático percibido. Se han obtenido elevados índices de consistencia interna (Tedeschi y Calhoun, 1996; So-kun Tan, 2007; Ho, Wing Chu y Yiu, 2008). Datos preliminares de validación de este instrumento en una muestra clínica española con TA y duelo complicado (Molés, Quero, Nebot, Rachyla y López, 2014) mostraron coeficientes altos de consistencia interna $(0,92)$ y de fiabilidad test-retest $(0,95)$. 


\section{Tratamiento}

Todos los participantes recibieron el protocolo de TA desarrollado por Botella, Baños y Guillén (2008). La duración de la intervención varió de 6 a 8 sesiones, dependiendo de la situación clínica de cada paciente. Cada una de las sesiones eran de 90 minutos de duración y se relizaban con periocidad semanal. Este protocolo incluye técnicas TCC y estrategias de psicología positiva. Los componentes terapéuticos incluidos en las sesiones fueron: psicoeducación, exposición, aceptación y elaboración del acontecimiento negativo y prevención de recaídas.

La mayoría de los participantes $(\mathrm{N}=66)$ recibieron el protocolo apoyado con $\mathrm{RV}$, mientras que 31 participantes recibieron el formato tradidional. Concretamente se utilizó «El mundo de EMMA» (véase la figura 1). Se trata de un sistema tridimensional que permite la manipulación de diferentes ambientes y símbolos a tiempo real y que acompañará al paciente durante todas las sesiones terapéuticas. Debido a esa flexibilidad respecto a la utilización de los diferentes símbolos, "El mundo de EMMA» permite ajustar el tratamiento a las características de cada paciente. El objetivo de «El mundo de EMMA» es ayudar a expresar y evocar los sentimiento y emociones de cada paciente, asociados al acontecimiento estresante, permitiendo así impulsar la superación del problema.

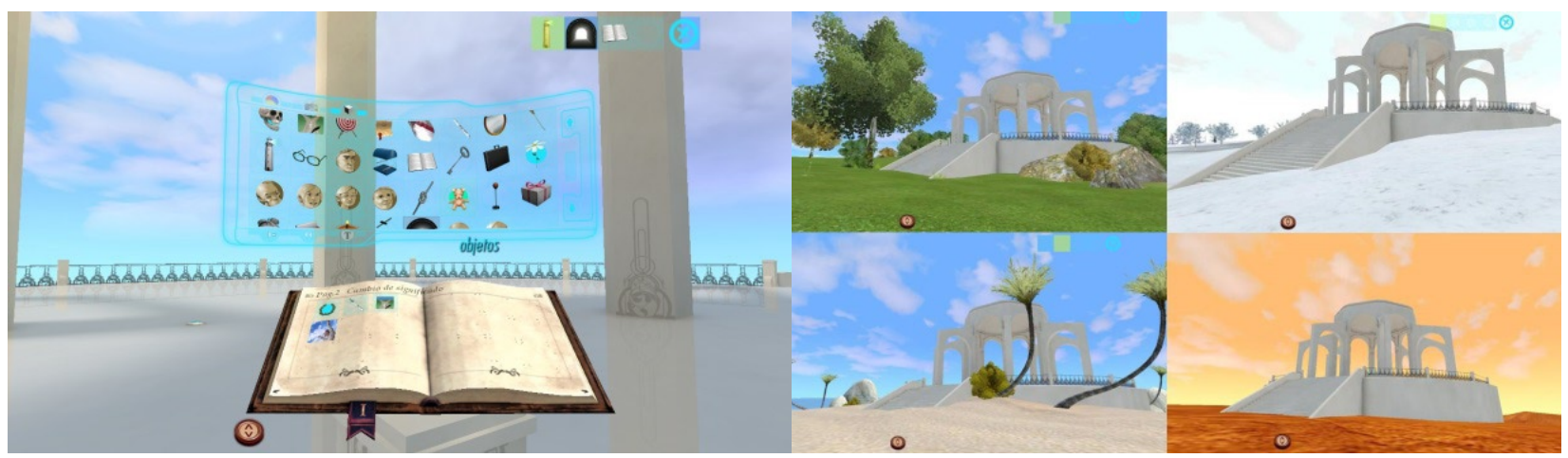

Figura 1. «El mundo de EMMA»

\section{Procedimiento}

Se recogieron datos de los paciente del SAP diagnosticados de TA referidos a las variables de comorbilidad con el eje II y de eficacia terapéutica anteriormente descritas. Atendiendo a la evaluación realizada con la SCID-II (First y cols., 1999), se divideron las participantes en dos grupos: grupo de TA con TP o rasgos patológicos de personalidad ( $N=55$ ) y grupo de TA $\sin$ TP $(N=42)$. Los datos relativos a la eficacia terapéutica se recogieron en dos momentos de evaluación: postratamiento y seguimiento a los tres meses. Después, se procedió a la realización de los análisis correspondientes mediante el Statistics Package for Social Sciences (SPSS22), versión en español. Todos los participantes formaron parte de un estudio de investigación previo en el que se estudiaba la eficacia de un tratamiento psicológico para el TA y firmaron el consentimiento informado participando de forma voluntaria. 


\section{Resultados}

En primer lugar, se realizó una prueba $t$ para muestras independientes con el objetivo de comprobar si había diferencias significativas en las medidas de eficacia entre los dos grupos experimentales antes del tratamiento. No se encontraron diferencias en ninguna de las medidas incluidas en este estudio (BDI, IEP, PTGI y PANAS). En la tabla 1 se presentan los estadísticos descriptivos para cada una de las medidas.

Tabla 1

Estadísticos descriptivos en el pretratamiento, postratamiento y seguimiento tres meses

\begin{tabular}{lcccccc}
\hline & \multicolumn{2}{c}{ M (DT) Pretratamiento } & \multicolumn{2}{c}{ M (DT) Postratamiento } & \multicolumn{2}{c}{ M (DT) Seg. 3 meses } \\
\cline { 2 - 7 } & TA+TP & TA & TA+TP & TA & TA+TP & TA \\
\hline \multirow{2}{*}{ BDI-II } & 23,13 & 21,67 & $9,69(7,45)$ & $7,43(6,34)$ & $7,45(6,51)$ & $6,08(5,20)$ \\
& $(10,61)$ & $(10,18)$ & & & & \\
IEP & 37,22 & 33,08 & 17,82 & $17,08(9,45)$ & $12,40(8,98)$ & $14,53(9,90)$ \\
& $(14,29)$ & $(11,05)$ & $(11,78)$ & & & 64,67 \\
\multirow{2}{*}{ PTGI } & 38,11 & 35,79 & 63,60 & 66,67 & 62,26 \\
\multirow{2}{*}{ PANAS } & $(22,54)$ & $(20,31)$ & $(22,21)$ & $(19,78)$ & $(22,54)$ & $(18,64)$ \\
& $26,83(6,95)$ & $25,07(8,26)$ & $18,48(5,93)$ & $16,25(4,63)$ & $16,62(5,16)$ & $16,73(5,76)$ \\
\hline
\end{tabular}

En segundo lugar, se calcularon los índices de cambio fiable (Jacobson y Truax, 1991) obtenidos para cada una de las medidas de eficacia (BDI, IEP, PTGI y PANAS) tanto en el postratamiento como en el seguimiento a los tres meses. Los porcentajes obtenidos en estas medidas en los dos momentos de evaluación se pueden ver en la tabla 2. Los resultados obtenidos tras aplciar la prueba Chi-cuadrado indicaron que en ninguna de las medidas de eficacia existían diferencias estadísticamente significativas entre ambos grupos ni en el postratamiento ni en el seguimiento.

Tabla 2

Cambio clínicamente significativo en cada una de las medidas de eficacia en el postratamiento $y$ en el seguimiento a los tres meses

\begin{tabular}{clcccc}
\hline & & \multicolumn{2}{c}{ Postratamiento } & \multicolumn{2}{c}{ Seguimiento tres meses } \\
\cline { 3 - 5 } & Recuperados & TA+TP & TA & TA+TP & TA \\
\hline \multirow{3}{*}{ bDI } & $44,89 \%$ & $52,5 \%$ & $13,46 \%$ & $23,07 \%$ \\
& Mejorados & $4,08 \%$ & $0 \%$ & $26,92 \%$ & $28,2 \%$ \\
& No cambio & $55,10 \%$ & $47,5 \%$ & $59,61 \%$ & $48,71 \%$ \\
& Empeorados & $0 \%$ & $0 \%$ & $0 \%$ & $0 \%$
\end{tabular}




\begin{tabular}{llcccc}
\hline & & \multicolumn{2}{c}{ Postratamiento } & \multicolumn{2}{c}{ Seguimiento tres meses } \\
\cline { 3 - 6 } & & TA+TP & TA & TA+TP & TA \\
\hline \multirow{3}{*}{ IEP } & recuperados & $68,75 \%$ & $66,66 \%$ & $81,57 \%$ & $80 \%$ \\
& Mejorados & $2,08 \%$ & $0 \%$ & $2,63 \%$ & $0 \%$ \\
& No cambio & $29,16 \%$ & $30,55 \%$ & $15,78 \%$ & $14,28 \%$ \\
& Empeorados & $0 \%$ & $2,77 \%$ & $0 \%$ & $5,71 \%$ \\
& recuperados & $13,46 \%$ & $23,08 \%$ & $23,25 \%$ & $15,78 \%$ \\
& Mejorados & $26,92 \%$ & $28,20 \%$ & $18,60 \%$ & $34,21 \%$ \\
& No cambios & $52,61 \%$ & $48,71 \%$ & $58,13 \%$ & $50 \%$ \\
& Empeorados & $0 \%$ & $0 \%$ & $0 \%$ & $0 \%$ \\
& recuperados & $46,15 \%$ & $55 \%$ & $42,76 \%$ & $54,05 \%$ \\
& Mejorados & $1,92 \%$ & $5 \%$ & $7,14 \%$ & $2,7 \%$ \\
& No cambio & $48,07 \%$ & $37,5 \%$ & $35,71 \%$ & $40,54 \%$ \\
& Empeorados & $3,84 \%$ & $2,5 \%$ & $2,38 \%$ & $2,7 \%$ \\
\hline
\end{tabular}

\section{Discusión y conclusiones}

El objetivo del presente estudio era explorar si existían diferencas en la eficacia del tratamiento del TA en pacientes con este problema y comorbilidad con TP o rasgos patológicos de personalidad y pacientes que solamente presentaban TA. Según los resultados obtenidos, no podemos concluir que existen diferencias significativas en la eficacia del tratamiento de los TA entre ambos grupos. Así pues, tanto los pacientes con TA comórbido con TP o rasgos patológicos como los pacientes con TA se beneficiaron de manera similar del tratamiento de los TA que habían recibido.

Nuestros datos no van en la linea de otras investigaciones previas que sugieren que presentar un TP o rasgo patológico influye de manera negativa en la eficacia de otros trastornos del eje I (Reich y Green, 1991). Sin embargo, sí se muestran similares a los resultados alcanzados en otros trabajos más recientes de revisión en los que se explora si la presencia de un TP afecta negativamente al tratamiento de los trastornos de ansiedad (Dressen y Arntz, 1998) y trastornos depresivos (Newton-Howes, 2008). Así, Dressen y Arntz (1998) no pueden concluir a partir de su revisión que la presencia de un TP influya negativamente en al eficacia del tratamiento de los trastornos de ansiedad. De forma similar, Newton-Howes (2008) no encontraron diferencias significativas en la eficacia del tratamiento del trastorno depresivo.

No hemos encontrado ningún estudio en la literatura hasta la fecha que explore la eficacia del tratamiento del TA cuando existe comorbilidad con un TP o con rasgos patológicos de personalidad. Por lo tanto, el presente trabajo es el primer estudio que explora la efiacia del tratamiento de los TA en pacientes con TP o rasgos.

No obstante, cabe destacar que, al tratarse de un estudio preliminar, hay que tener en cuenta diversas limitaciones que han podido influir en los resultados. Por un lado, está el pequeño tamaño de la muestra de pacientes. Por otro lado, no se realizó una evaluación independiente para confirmar el diagnóstico de TP o la presencia de rasgos patológicos de personalidad. Además, en el grupo de TA con TP se agruparon tanto a los pacientes con un TP como 
a pacientes que solo presentaban rasgos patológicos, pudiendo ser uno de los factores que quizás haya tendido una mayor influencia en los resultados obtenidos. Y, por último, tampoco están claramente especificado en el estudio los tipos de rasgos patológicos que presentaban los pacientes. No obstante, a pesar de las limitaciones, los resultados obtenidos podrían aportar un nuevo marco de investivacion en lo que respecta a los TA.

En conclusión, los resultados preliminares obtenidos parecen indicar que la presencia de un TP o rasgos patológicos de la personalidad no influyen en la eficacia del tratamiento para los TA y tampoco en el mantenimiento de los cambios a medio plazo. Por lo tanto, ambos grupos parecen beneficiarse por igual de un tratamiento TCC en formato tradicional o apoyado en la RV. No obstante, teniendo en cuenta la relevancia de los TA en la actualidad, junto con la escasa investigación que hay tanto sobre su tratamiento como sobre el papel de los TP en la eficacia del mismo, es necesario seguir investigando en esta línea y llevar a cabo estudios que superen las limitaciones citadas anteriormente. Estos estudios ayudarán a clarificar el papel de los TP o rasgos patológicos de personalidad en la eficacia de tramiento, permitiendo variar y, en consecuencia, mejorar el tratamiento administrado a los pacientes en función de si presentan o no comorbilidad con TP.

\section{Referencias bibliográficas}

American Psychiatric Association (APA). (2000). Diagnostic and statical manual of mental disorders DSM-IV-TR (4th ed. Text revision). Washington, DC: APA.

American Psychiatric Association (APA). (2013). Diagnostic and statical manual of mental disorders DSM-V (5th ed.). Washington, DC: APA.

Andreu-Mateu, S., Botella, C., Quero, S., Guillén, V. y Baños, R. (2012). La utilización de la realidad virtual y estrategias de psicología positiva en el tratamiento de los trastornos adaptativos. Psicología Conductual, 20, 323-348.

Baños, R. M., Botella, C., Guillén, V., García-Palacios, A., Jorquera, M. y Quero, S. (2008). Un programa de tratamiento para los trastornos adaptativos. Un estudio de caso. Apuntes de Psicología, 26, 303-316.

Baños, R. M., Guillén, V., Quero, S., García-Palacios, A., Alcaniz, M. y Botella, C. (2011). A virtual reality system for the treatment of stress-related disorders: A preliminary analysis of efficacy compared to a standard cognitive behavioral program. International Journal of Human-Computer Studies, 69, 602-613.

Beck, A. T., Steer, R. A, y Brown, G. K. (1996). Manual for the Beck Depression Inventory-II. San Antonio, TX: Psychological Corp.

Ben-Itzhak, S., Bluvstein, I., Schreiber, S., Aharonov-Zaig, I., Maor, M., Lipnik, R. y Bloch, M. (2012). The Effectiveness of Brief Versus Intermediate Duration Psychodynamic Psychotherapy in the Treatment of Adjustment Disorder. Journal of Contemporary Psychotherapy, 42, 249-256.

Black, D. W., Wesner, R. B., Gabel, J., Bowers, W. y Monahan, P (1994). Predictors of shortterm treatment response in 66 patients with panic disorder. Journal of Affective Disorders, 30, 233-241.

Botella, C., Baños, R. M. y Guillén, V. (2008). Una propuesta de tratamiento para los trastornos adaptativos: creciendo en la adversidad. En C. Vázquez y G. Hervás (eds.), Psicología positiva aplicada (pp. 129-154). Bilbao: Desclée de Brouwer.

Botella, C., Osma, J., García Palacios, A., Guillén, V. y Baños, R. M. (2008). Treatment of complicated grief using virtual reality. A case report Death Studies, 32, 674-692. 
Calvo, E. (2009). Duración de la incapacidad temporal asociada a diferentes patologías en trabajadores españoles. Recuperado de http://www.seg-social.es/prdi00/groups/public/ documents/binario/146662.pdf

Carta, M. G., Balestrieri, M., Murru. A. y Hardoy, M. C. (2009). Adjustment Disorder: epidemiology, diagnosis and treatment. Clinical Practice and Epidemiology in Mental Health, 5, $1-15$.

Casey, P., Dowrick, C. y Wilkinson, G. (2001). Adjustment disorders. Fault line in the pychiatric glossary. The British Journal of Psychiatry, 179, 479-481.

Casey, P., Jabbar, F., O'Leary, E., y Doherty, A. M.(2014). Suicidal behaviours in adjustment disorder and depressive episode. Journal of Affective Disorders, 174, 441-446.

Doherty, A. M., Jabbar, F., Kelly, B. D. y Casey, P. (2014). Distinguishing between adjustment disorder and depressive episode in clinical practice: The role of personality disorder. Journal of Affective Disorders, 168, 78-85.

Dreessen, L. y Arntz, A. (1998). The impact of personality disorders on treatment outcome of anxiety disorders: Best evidence synthesis. Behaviour Research and Therapy, 36, 483504.

Echeburúa, E., Corral, P. y Fernández-Montalvo, J. (2000). Escala de Inadaptación: Propiedades psicométricas en contextos clínicos. Análisis y Modificación de Conducta, 26, 325340.

First, M. B., Spritzer, R. L., Gibbon, M. y Williams, J. B. (1999). Entrevista Clínica Estructurada para los Trastornos del Eje II del DSM-IV, Versión Clínica. Barcelona: MASSON.

Hsiao, F. H., Lai, Y. M., Chen, Y. T., Yang, T. T., Liao, S. C., Ho, R. T. H., Ng, S. M., Chang, C. L y Jow, G. M. (2014). Efficacy of psychotherapy on diurnal cortisol patterns and suicidal ideation in adjustment disorder with depressed mood. General Hospital Psychiatry, 36, 214-219.

Jacobson, N. S. y Truax, P. (1991). Clinical significance: A statistical approach to defining meaningful change in psychotherapy research. Journal of Consulting and Clinical Psychology, 59, 12-19.

Molés, M., Quero, S., Nebot, S., Rachyla, I. y López, M. T. (2014). Datos preliminares de validación del Inventario de Crecimiento Postraumático en población clínica española con trastornos relacionados con el estrés. Poster presentado en el II Congreso Nacional de Psicología Positiva. Oropesa, España.

Newton-Howes, G. (2008). Influencia de los trastornos de la personalidad en el pronóstico de la depresión. Psychiatry, 7, 105-108.

Organización Mundial de la Salud (1992). Clasificación Internacional de Enfermedades (CIE10) (10. ${ }^{a}$ edición). Madrid: Meditor.

Pirgerson, H. G., Maciejewsku, P. K., Reynolds, C. F., Bierhals, A. J., Newsom, J. T., Fasiczka, A. y cols. (1995). Inventory of Complicated Grief. A scale to measure maladaptative symptoms of loss. Psychiatry Research, 59, 65-79.

Quero, S., Andreu-Mateu, S., Moragrega, I., Baños, R. M., Molés, M., Nebot, S. y Botella, C. (en prensa). Un programa cognitivo-conductual que utiliza la realidad virtual para el tratamiento de los trastornos adaptativos: una serie de casos. Revista Argentina de Psicología Clínica.

Quero, S., Molés, M., Mor, S., Baños, R. M. y Botella, C. (2014). Spanish validation of the complicated grief inventory adapted for adjustment disorders. ABCT 48th Annual Convention.

Reich, J. H. y Green, A. I. (1991). Effect of personality disorder on outcome of treatment. The Journal of Nervous and Mental Disease, 179, 74-82. 
Reich, J. H. y Vasile, R. G. (1993). Effect of personality disorders on the treatment outcome of axis I conditions: an update. The Journal of Nervous and Mental Disease, 181, 475484.

Sanz, J., Navarro, M. E. y Vázquez, C. (2003). Adaptación española del Inventario para la Depresión de Beck-II (BDI-II): propiedades psicométricas en estudiantes universitarios. Análisis y modificación de conducta, 29, 239-288.

Srivastava, M., Talukdar, U. y Lahan, V. (2011). Meditation for the management of adjustment disorder anxiety and depression. Complementary Therapies in Clinical Practice, 17, 241245.

Tedeschi, R. G. y Calhoun, L. G. (1996). The Posttraumatic Growth Inventory: Measuring the Positive Legacy of Trauma. Journal of Traumatic Stress, 9, 455-471.

Watson, D., Clark, L. A. y Tellengen, A. (1988). Development and validation of brief measures of positive and negative affect: the PANAS scales. Journal of Personality and Social Psychology, 54, 1063-1070. 